\title{
Reconstruction of annular bi-layered media in cylindrical waveguide section
}

\author{
Anders Eriksson ${ }^{1}$, Larisa Beilina ${ }^{2 *}$ and Truls Martin Larsen ${ }^{3}$
}

"Correspondence:
larisa@chalmers.se
${ }^{2}$ Department of Mathematical
Sciences, Chalmers University of
Technology and University of
Gothenburg, Gothenburg, 41296,
Sweden
Full list of author information is
available at the end of the article

available at the end of the article

\begin{abstract}
We consider a radial transverse resonance model for a circular cylindrical waveguide composed into two layers with different frequency dependent complex dielectric constants. An inverse problem with four unknowns - three physical material parameters and one dimensional dielectric layer thickness parameter - is solved by employing TE1 10 and TE210 modes with different radial field distribution. First the resonance frequencies and quality factors are found fitting a Lorentzian function to the 'measured' data, using the method of least squares. Then found resonance frequencies and quality factors are used in a second inverse Newton-Raphson algorithm which solves four transverse resonance equations in order to get four unknown parameters. The use of TE110 and TE210 models offers one-dimensional radial tomographic capability. An open ended coaxial waveguide quarter-wave resonator is added to the sensor topology, and the effect on the convergence of numerical method is investigated.
\end{abstract}

Keywords: reconstruction of material parameters in a waveguide; transverse resonance model; open ended coaxial waveguide resonator; least squares problem

\section{Introduction}

Extraction of material parameters and/or dimensions based on distributed resonator measurements has been around for decades. Characterization of distributed microwave resonators dielectric material from resonance frequency and quality factor measurements is found in [1]. A comparison of inverse methods for extracting resonant frequency and quality factor is given in [2]. Typically, the dielectric filling of the resonators is homogeneous, but there are not really any restrictions for allowing inhomogeneous dielectric filling.

In this work, we model two annular concentric cylindrical layers which are enclosed in a finite conductive metallic pipe, each with a frequency dependent dielectric constant, see Figure 1. We develop reliable least squares algorithms which allow determine four unknown physical parameters using a transverse resonance radial model, with additional model of the open ended coaxial waveguide quarter wave resonators. The possible applications are characterization of metallic pipes with annular flow, or characterization of optical fibers in similar geometries.

In this work we use the Bruggemans model for emulsion permittivity $[3,4]$. This model is applied for saline water mixed with oil as well as for liquid droplets in gas. For the latter, the Bruggemans model is used twice - first for water mixed with oil in liquid droplets, and

(c) The Author(s) 2017. This article is distributed under the terms of the Creative Commons Attribution 4.0 International License (http://creativecommons.org/licenses/by/4.0/), which permits unrestricted use, distribution, and reproduction in any medium, provided you give appropriate credit to the original author(s) and the source, provide a link to the Creative Commons license, and indicate if changes were made. 
then again to calculate the effective permittivity of liquid droplets in gas. Several models for effective complex permittivity of metal powders in insulating dielectrics (e.g. teflon) are studied in [4], and one conclusion is that the Bruggemans model is relatively accurate for predicting of the real part of permittivity, while the well-known Maxwell-Garnett model [4] have higher accuracies for the imaginary part. This is relevant for the case of an oil continuous regime with water content of high salinity. In this case the electrically conducting saline water droplets are comparable to electrically conducting metal powder. Another popular model - the Gadanis model for saline water [5] - we use to determine a complex permittivity of the saline water. In this model the complex permittivity is a function of salinity $(s)$, temperature $(T)$ and frequency $(f)$.

Thus, by applying these two permittivity models with corresponding fractions for each medium to our problem (assuming that the known chemical substances are presented but with unknown ratios), the parameters are reduced to four unknowns. In the case of using directly frequency-dependent complex permittivity for the liquid, and another frequencydependent complex permittivity for the gas, four unknowns would be left to find just for one frequency point along with the liquid thickness. Then from these permittivities, the water liquid ratio (WLR) and droplet gas ratio (DGR) can be found.

\section{Model equations for cylindrical waveguides}

Let $G \subset \mathbb{R}^{n}, n=1,2,3$ be a bounded domain with a piecewise smooth boundary $\partial G$ and $x$ be a point in this domain. Let $\Omega \subset G$ be bounded subdomain representing our waveguide with a boundary $\partial \Omega$. As a model problem we have used a full wave RF resonance model of [1] using transverse resonance method. This model is very computationally efficient and compact (equation wise). It can model infinite long cylindrical pipe or waveguide, filled with arbitrary concentric layers.

We consider source free Maxwell's equations for the electric $E:=E(x, \omega)$ and magnetic $H:=H(x, \omega)$ fields in the form

$$
\begin{aligned}
& \nabla \times E=-i \omega \mu H, \\
& \nabla \times H=i \omega \varepsilon E,
\end{aligned}
$$

with appropriate initial and boundary conditions. Here, $\omega$ is the frequency, $\varepsilon, \mu$ are the complex dielectric permittivity and dielectric permeability constants, respectively, defined as

$$
\begin{aligned}
& \varepsilon=\varepsilon_{0} \varepsilon_{r}, \\
& \mu=\mu_{0} \mu_{r},
\end{aligned}
$$

where $\varepsilon_{0}$ is the electric permittivity in vacuum, $\varepsilon_{r}$ is the dimensionless relative electric permittivity, $\mu_{0}$ is the magnetic permeability in vacuum, $\mu_{r}$ is the dimensionless relative magnetic permeability. The complex dielectric constant can be expressed as:

$$
\varepsilon=\varepsilon_{0} \varepsilon_{r}=\varepsilon_{0}\left(\varepsilon_{\mathrm{Re}}-i \varepsilon_{\mathrm{Im}}\right)=\varepsilon_{0}\left(\varepsilon_{\mathrm{Re}}-i \frac{\sigma}{\omega}\right),
$$

where $\varepsilon_{\mathrm{Re}}, \varepsilon_{\mathrm{Im}}$ are the real and imaginary parts of the relative permittivity for an arbitrary material, respectively, $\sigma$ is the electrical conductivity, and $\omega$ is angular frequency. The 
equation (3) applies to imperfect metal with finite conductivity as well as to the dielectric materials under investigation.

The radial propagation constant is defined as

$$
\beta^{2}=k^{2}-k_{z}^{2}
$$

where

$$
k^{2}=\omega^{2} \mu_{0} \mu_{r} \varepsilon_{0} \varepsilon_{r}
$$

is a free space wave number and $k_{z}$ is the longitudinal wavenumber, which includes a complex resonance frequency

$$
\omega_{0}=\omega_{0 \mathrm{Re}}+i \omega_{0 \mathrm{Im}}
$$

at $\omega \rightarrow \omega_{0}$.

We rewrite system (1) in cylindrical coordinates $(r, \varphi, z)$ for $E=\left(E_{r}, E_{\varphi}, E_{z}\right)$ and $H=$ $\left(H_{r}, H_{\varphi}, H_{z}\right)$ to get

$$
\begin{aligned}
& i \omega \varepsilon E_{r}=\frac{1}{r} \frac{\partial H_{z}}{\partial \varphi}+i \beta H_{\varphi}, \\
& i \omega \varepsilon E_{\varphi}=-i \beta H_{r}-\frac{\partial H_{z}}{\partial r}, \\
& i \omega \varepsilon E_{z}=\frac{1}{r}\left(\frac{\partial\left(r H_{\varphi}\right)}{\partial r}-\frac{\partial H_{r}}{\partial \varphi}\right), \\
& -i \omega \mu H_{r}=\frac{1}{r} \frac{\partial E_{z}}{\partial \varphi}+i \beta E_{\varphi}, \\
& -i \omega \mu H_{\varphi}=-i \beta E_{r}-\frac{\partial E_{z}}{\partial r}, \\
& -i \omega \mu H_{z}=\frac{1}{r}\left(\frac{\partial\left(r E_{\varphi}\right)}{\partial r}-\frac{\partial E_{r}}{\partial \varphi}\right) .
\end{aligned}
$$

We note that often the solutions of (7) is efficient represent in the terms of Hankels functions $H_{m}^{(1)}, H_{m}^{(2)}$ of the first and second order, see [6], and these solutions are given in [7]. In Sections 4.1, 4.2 we use both Hankels and Bessels functions. Below we present solutions of (7) in a cylindrical waveguides for TE and TM modes expressed via Bessels functions.

\subsection{TE modes in a cylindrical waveguide}

For transverse electric (TE) mode we assume that $E_{z}=0$ in (7) and the longitudinal magnetic field $H_{z}$ satisy the Helmholtz equation

$$
\triangle H_{z}+k^{2} H_{z}=0
$$

where $\triangle$ is the Laplace operator in cylindrical coordinates, $k^{2}$ is defined in (5) and

$$
H_{z}(r, \varphi, z):=H_{z}(r, \varphi) e^{-i \beta z}
$$


Inserting the above equation into (8) we obtain

$$
\frac{\partial^{2} H_{z}(r, \varphi)}{\partial r^{2}}+\frac{1}{r} \frac{\partial H_{z}(r, \varphi)}{\partial r}+\frac{1}{r^{2}} \frac{\partial^{2} H_{z}(r, \varphi)}{\partial \varphi^{2}}+k_{z}^{2} H_{z}(r, \varphi)=0
$$

where $k_{z}$ can be computed from (4). The function $H_{z}(r, \varphi)$ can be further represented as

$$
H_{z}(r, \varphi)=R(r) \Phi(\varphi)
$$

Inserting (11) into (10) we obtain two partial differential equations for functions $R(r), \Phi(\varphi)$, respectively, which can be solved explicitly, see details on [8]. Then the function $H_{z}(r, \varphi)$ can be computed as

$$
H_{z}(r, \varphi)=J_{n}\left(k_{z} r\right)(A \sin (n \varphi)+B \cos (n \varphi)) e^{-i \beta z},
$$

where $J_{n}\left(k_{z} r\right)$ is the Bessel function of the first kind of order $n$, see [6].

Thus, taking $E_{z}=0$ in (7) and using (12) we can write the resulting equations for TE modes:

$$
\begin{aligned}
& E_{r}(r, \varphi, z)=-\frac{i \omega \mu}{k_{z}^{2} r} \frac{\partial H_{z}}{\partial \varphi}=-\frac{i \omega \mu n}{k_{z}^{2} r} J_{n}\left(k_{z} r\right)(A \cos (n \varphi)-B \sin (n \varphi)) e^{-i \beta z} \\
& E_{\varphi}(r, \varphi, z)=\frac{i \omega \mu}{k_{z}^{2}} \frac{\partial H_{z}}{\partial r}=\frac{i \omega \mu}{k_{z}^{2}} J_{n}^{\prime}\left(k_{z} r\right)(A \sin (n \varphi)+B \cos (n \varphi)) e^{-i \beta z} \\
& H_{r}(r, \varphi, z)=-\frac{i \beta}{k_{z}^{2}} \frac{\partial H_{z}}{\partial r}=-\frac{i \beta}{k_{z}^{2}} J_{n}^{\prime}\left(k_{z} r\right)(A \sin (n \varphi)+B \cos (n \varphi)) e^{-i \beta z} \\
& H_{\varphi}(r, \varphi, z)=-\frac{i \beta}{k_{z}^{2} r} \frac{\partial H_{z}}{\partial \varphi}=-\frac{i \beta n}{k_{z}^{2} r} J_{n}\left(k_{z} r\right)(A \cos (n \varphi)-B \sin (n \varphi)) e^{-i \beta z}
\end{aligned}
$$

where $J_{n}^{\prime}\left(k_{z} r\right)$ denotes the derivative of the Bessel function of the first kind with respect to $r$ which can be computed as

$$
J_{n}^{\prime}\left(k_{z} r\right)=\frac{\partial\left(J_{n}\left(k_{z} r\right)\right)}{\partial r}=k_{z} \frac{\partial J_{n}\left(k_{z} r\right)}{\partial\left(k_{z} r\right)} .
$$

\subsection{TM modes in a cylindrical waveguide}

For transverse magnetic (TM) mode we assume that $H_{z}=0$ in (7) and the longitudinal electric field $E_{z}$ satisy the Helmholtz equation

$$
\triangle E_{z}+k^{2} E_{z}=0
$$

where $\triangle$ is again the Laplace operator in cylindrical coordinates, $k^{2}$ is defined in (5) and

$$
E_{z}(r, \varphi, z):=E_{z}(r, \varphi) e^{-i \beta z}
$$

Inserting the above equation into (15) we obtain

$$
\frac{\partial^{2} E_{z}(r, \varphi)}{\partial r^{2}}+\frac{1}{r} \frac{\partial E_{z}(r, \varphi)}{\partial r}+\frac{1}{r^{2}} \frac{\partial^{2} E_{z}(r, \varphi)}{\partial \varphi^{2}}+k_{z}^{2} E_{z}(r, \varphi)=0 .
$$


The function $E_{z}(r, \varphi)$ can be further represented as

$$
E_{z}(r, \varphi)=R(r) \Phi(\varphi)
$$

Inserting (18) into (17) we obtain two partial differential equations for functions $R(r), \Phi(\varphi)$, respectively, which can be solved explicitly, see details on [8]. Then the function $E_{z}(r, \varphi)$ can be computed as

$$
E_{z}(r, \varphi)=J_{n}\left(k_{z} r\right)(A \sin (n \varphi)+B \cos (n \varphi)) e^{-i \beta z},
$$

where $J_{n}\left(k_{z} r\right)$ is the Bessel function of the first kind of order $n$, see [6].

Now taking $H_{z}=0$ in (7) and using (19) we can write the resulting equations for TM modes:

$$
\begin{aligned}
& E_{r}(r, \varphi, z)=-\frac{i \beta}{k_{z}^{2}} \frac{\partial E_{z}}{\partial r}=-\frac{i \beta}{k_{z}^{2}} J_{n}^{\prime}\left(k_{z} r\right)(A \sin (n \varphi)+B \cos (n \varphi)) e^{-i \beta z} \\
& E_{\varphi}(r, \varphi, z)=-\frac{i \beta}{k_{z}^{2} r} \frac{\partial E_{z}}{\partial \varphi}=-\frac{i \beta n}{k_{z}^{2} r} J_{n}\left(k_{z} r\right)(A \cos (n \varphi)-B \sin (n \varphi)) e^{-i \beta z} \\
& H_{r}(r, \varphi, z)=\frac{i \omega \varepsilon}{k_{z}^{2} r} \frac{\partial E_{z}}{\partial \varphi}=\frac{i \omega \varepsilon n}{k_{z}^{2} r} J_{n}\left(k_{z} r\right)(A \cos (n \varphi)-B \sin (n \varphi)) e^{-i \beta z} \\
& H_{\varphi}(r, \varphi, z)=-\frac{i \omega \varepsilon}{k_{z}^{2}} \frac{\partial E_{z}}{\partial r}=-\frac{i \omega \varepsilon}{k_{z}^{2}} J_{n}^{\prime}\left(k_{z} r\right)(A \sin (n \varphi)+B \cos (n \varphi)) e^{-i \beta z}
\end{aligned}
$$

\section{Statement of inverse problem}

We consider the inverse problem for two annular cylindrical layers with outer boundary which is a perfect electrical conductor (PEC), see Figure 1. In this figure, the region between $R_{0}$ and $R_{1}$ is filled with a finite electrical conductor, $R_{1}-R_{2}$ is thickness of a liquid layer of a waveguide with dielectric permittivity $\varepsilon_{1}:=\varepsilon_{1}\left(R_{\mathrm{WLR}}, s\right), R_{2}$ is radius of a waveguide with dielectric permittivity $\varepsilon_{2}:=\varepsilon_{2}\left(R_{\mathrm{DGR}}, s\right)$. Here, parameters $R_{\mathrm{WLR}}, R_{\mathrm{DGR}}, s$ are described in Table 1.

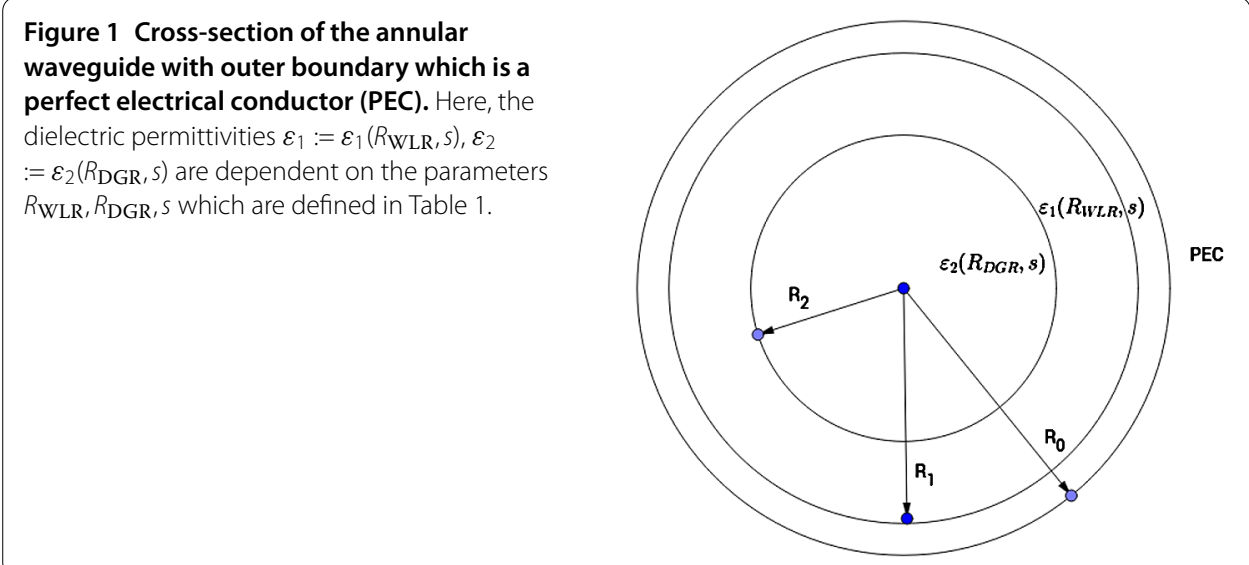


Table 1 Parameters to be determined in IP

\begin{tabular}{ll}
\hline Parameter & Description \\
\hline$h$ & Thickness of liquid layer - liquid having arbitrary mixture ratio (Water Liquid Ration) of \\
& condensate and saline water. \\
$R_{\text {WLR }}$ & Water Liquid Ratio (WLR) - water fraction in liquid. \\
$R_{\text {DGR }}$ & Droplet Gas Ratio (DGR) - ratio of liquid immersed in droplet form in gas continuous volume \\
& (note that WLR and Salinity is the same in liquid film as well as in droplets). \\
$s$ & Salinity - salt concentration in water. \\
\hline
\end{tabular}

The dielectric permittivities $\varepsilon_{1}, \varepsilon_{2}$ are such that

$$
\begin{aligned}
& \left(\varepsilon_{1}, \varepsilon_{2}\right)=\left(\varepsilon_{0} \varepsilon_{r 1}, \varepsilon_{0} \varepsilon_{r 2}\right)=\text { const. for } x \in \bar{\Omega}, \\
& \varepsilon_{1} \in\left[\varepsilon_{1 \min }, \varepsilon_{1 \max }\right], \quad \operatorname{Re}\left(\varepsilon_{1}\right) \geq 1.0, \quad \operatorname{Im}\left(\varepsilon_{1}\right) \leq 0.0, \\
& \varepsilon_{2} \in\left[\varepsilon_{2 \min }, \varepsilon_{2 \max }\right], \quad \operatorname{Re}\left(\varepsilon_{2}\right) \geq 1.0, \quad \operatorname{Im}\left(\varepsilon_{2}\right) \leq 0.0 .
\end{aligned}
$$

We assume that magnetic permeability in every layer is such that $\left(\mu_{1}, \mu_{2}\right)=\left(\mu_{0} \mu_{r 1}, \mu_{0} \mu_{r 2}\right)$ with known relative magnetic permeability in every layer $\mu_{r 1}=\mu_{r 2}=1$.

Our inverse problem is following:

Inverse Problem (IP) Under the condition that there are no water vapor in consideration, determine the four different parameters: salt $s(\mathrm{NaCl})$, hydrocarbon gas $R_{\mathrm{DGR}}$, water $R_{\mathrm{WLR}}$ and the thickness of liquid layer parameter $h$, see Table 1 , assuming that the observed reflection coefficient $S_{11}$ in an open ended coaxial waveguide quarter wave resonator (see Section 5) is known for all frequencies on the frequency interval $[\underline{\omega}, \bar{\omega}]$ :

$$
\left.S_{11}(r, \omega)\right|_{r=r_{\text {obs }}}=\left.\tilde{S}_{11}(r, \omega)\right|_{r=r_{\text {obs }}}, \quad \forall \omega \geq \underline{\omega}=\text { const. }>0 .
$$

In our IP, the function $\left.\tilde{S}_{11}(r, \omega)\right|_{r=r_{\text {obs }}}$ in (22) represents measurements at the point $r=r_{\text {obs }}$ for all physical frequencies on the frequency interval $[\underline{\omega}, \bar{\omega}]$.

\section{Radial transverse resonance method}

In [7] was shown that only for the angular index $m=0$, pure TE or pure TM modes can accommodate in a two-layer dielectric cylindrical waveguide. However, it is trivial to show that if also the longitudinal wave-number $k_{z}$ is zero, pure TE and TM modes can accommodate. We note that $k_{z}=0$ is equivalent to pure radial resonance.

The quality factor of one of the two TE or TM modes will in theory be decreased by leaking waves, e.g. surface waves. Typically, for transmission based resonator measurements, the external coupling to the resonator is relatively weak - thus, $\left|S_{11}\right|^{2}+\left|S_{21}\right|^{2} \approx 1$, indicating that the unloaded quality factor $Q_{0}$ (stored energy to dissipated energy ratio) is dominant. In practice, this is realized by relatively small antennas/probes such that mainly fringing antenna field is exciting the resonance. Under such conditions, external quality factor is relatively large compared to the unloaded quality factor, rendering unloaded $Q$ (intrinsic desired resonator $Q$ ) equal to the loaded $Q$ (measured $Q$ ). The $Q$-factor of the composed multi-concentric layered structure is computed as in [9]

$$
Q_{0}=\frac{\omega_{0 \mathrm{Re}}}{2 \omega_{0 \mathrm{Im}}},
$$


where $\omega_{0 \mathrm{Re}}, \omega_{0 \mathrm{Im}}$ are given in (6). Setting the radial propagation constant $v:=\beta=\sqrt{k^{2}-k_{z}^{2}}$, and assuming that there is no variation in $z$-direction (i.e. a pure radial resonance) renders the propagation constant as $v=\omega \sqrt{\mu \varepsilon}$ with $\mu \varepsilon=\mu_{0} \mu_{r} \varepsilon_{0} \varepsilon_{r}$.

Let us now establish a suitable radial impedance transformation model. Since the wave impedance is anisotropic in radial direction [7], the reflection coefficient must be derived accordingly. Let $Y_{C}^{+}$and $Y_{C}^{-}$be the anisotropic admittances in outward and inward directions, respectively. We define the reflection at the load as $\Gamma=V^{-} / V^{+}$, where $V^{-}, V^{+}$are reflected and incident voltage at load $Z_{L}$, respectively, the outgoing current as $I^{+}=Y_{C}^{+} V^{+}$ and the reflected current as $I^{-}=Y_{C}^{-} V^{-}$. Finally, we define $V_{L}:=V^{+}+V^{-}, I_{L}:=I^{+}-I^{-}$which renders a reflection coefficient at the load impedance $Z_{L}$ :

$$
\Gamma_{L}=\frac{Z_{C}^{-}\left(Z_{L}-Z_{C}^{+}\right)}{Z_{C}^{+}\left(Z_{L}+Z_{C}^{-}\right)}
$$

where characteristic anisotropic impedances $Z_{C}^{-}, Z_{C}^{+}$are defined as $Z_{C}^{-}=\left(Y_{C}^{-}\right)^{-1}$ and $Z_{C}^{+}=$ $\left(Y_{C}^{+}\right)^{-1}$, respectively. We note that equation (24) simplifies to:

$$
\Gamma_{L}=\frac{Z_{L}-Z_{C}}{Z_{L}+Z_{C}}
$$

for isotropic characteristic impedance.

\subsection{Impedance transformation for TE wave}

For cylindrical TE wave the characteristic impedance $Z_{\text {Cout }}$ for a radial outgoing TE wave [7] is given by the formula:

$$
Z_{\text {Cout }}=\frac{E_{\varphi}}{H_{z}}=i \frac{\omega \mu H_{m}^{(2) \prime}(v r)}{v H_{m}^{(2)}(v r)},
$$

where $E_{\varphi}, H_{z}$ are defined in system (7), $H_{m}^{(2)}$ is the Hankel function of the second kind and $H_{m}^{(2) \prime}$ is the derivative of the Hankel function of the second kind, see [6].

The characteristic impedance for a radial incoming wave is defined as

$$
Z_{\mathrm{Cin}}=\frac{E_{\varphi}}{H_{z}}=-i \frac{\omega \mu H_{m}^{(1) \prime}(v r)}{\nu H_{m}^{(1)}(v r)},
$$

where $E_{\varphi}, H_{z}$ are defined in system (7), $H_{m}^{(1)}$ is the Hankel function of the first kind and $H_{m}^{(1) \prime}$ is the derivative of the Hankel function of the first kind, see [6].

With the voltage ratio $\frac{V_{0}^{-}}{V_{0}^{+}}$at the load impedance at $r_{0}$ defined as

$$
\frac{V_{0}^{-}}{V_{0}^{+}}=\frac{H_{m}^{(2) \prime}(v r) Z_{\text {Cin }}\left(r_{0}\right)\left(Z_{L}-Z_{\text {Cout }}\left(r_{0}\right)\right)}{H_{m}^{(1) \prime}(v r) Z_{\text {Cout }}\left(r_{0}\right)\left(Z_{L}+Z_{\text {Cin }}\left(r_{0}\right)\right)}
$$

the transformed load impedance from $r_{0}$ to $r$ becomes

$$
Z_{\text {out }}(r)=\frac{Z_{\mathrm{Cin}}(1+\Gamma(r))}{\frac{Z_{\mathrm{Cin}}}{Z_{\mathrm{Cout}}}-\Gamma(r)}=\frac{Z_{\mathrm{Cin}}\left(1+\frac{V_{0}^{-} H_{m}^{(1) \prime}(v r)}{V_{0}^{+} H_{m}^{(2) \prime}(v r)}\right)}{\frac{Z_{\mathrm{Cin}}}{Z_{\mathrm{Cout}}}-\frac{V_{0}^{-} H_{m}^{(1)}(v r)}{V_{0}^{+} H_{m}^{(2) \prime}(v r)}}
$$


We simplify last expression to get numerically efficient formulas which are used in computations by introducing notations

$$
\begin{aligned}
& F_{1}(x)=Z_{L} H_{m}^{(2)}(x)-B H_{m}^{(2) \prime}(x), \\
& F_{2}(x)=B H_{m}^{(1) \prime}(x)-Z_{L} H_{m}^{(1)}(x),
\end{aligned}
$$

where the load $Z_{L}$ is given at radius $r_{0}\left(\right.$ for $r_{0}>r$ ). Then (29) can be rewritten as

$$
Z_{\text {out }}(r)=\frac{B H_{m}^{(1) \prime}(v r) F_{1}\left(v r_{0}\right)+H_{m}^{(2) \prime}(v r) F_{2}\left(v r_{0}\right)}{H_{m}^{(1)}(v r) F_{1}\left(v r_{0}\right)+H_{m}^{(2)}(v r) F_{2}\left(v r_{0}\right)},
$$

with $B=\frac{i \omega \mu}{v}=i \sqrt{\frac{\mu}{\varepsilon}}$. We scale this expression with exponential function in order to handle finite metal conductivity:

$$
Z_{\text {out }}(r)=B \cdot \frac{e^{2 i v\left(r-r_{0}\right)} H_{m}^{(1) \prime}(v r) F_{1}\left(v r_{0}\right)+H_{m}^{(2) \prime}(v r) F_{2}\left(v r_{0}\right)}{e^{2 i v\left(r-r_{0}\right)} H_{m}^{(1)}(v r) F_{1}\left(v r_{0}\right)+H_{m}^{(2)}\left(v r_{0}\right) F_{2}\left(v r_{0}\right)} .
$$

The inward input impedance into the innermost region is defined as

$$
Z_{\text {in }}(r)=B \frac{J_{m}^{\prime}(v r)}{J_{m}(v r)},
$$

where $J_{m}$ is the Bessel function $J_{m}^{\prime}$ is the derivative of the Bessel function of the first kind, see [6]. Resonance condition is fulfilled when $Z_{\text {in }}\left(r_{\text {bound }}\right)+Z_{\text {out }}\left(r_{\text {bound }}\right)=0$ for both real and imaginary parts. The resonance condition can be calculated at any radius $r_{\text {bound }}$ inside the circular cylindrical region. In this work $r_{\text {bound }}=R_{2}$, see Figure 1 .

\subsection{Impedance transformation for cylindrical TM wave}

We follow the same steps as for TE wave, except that the characteristic impedance for an outgoing wave is given by the formula:

$$
Z_{\text {Cout }}=-\frac{E_{z}}{H_{\phi}}=-\frac{i v H_{m}^{(2)}(v r)}{\varepsilon k H_{m}^{(2)}(v r)} .
$$

The characteristic impedance for an incoming wave can be computed as

$$
Z_{\mathrm{Cin}}=-\frac{E_{z}}{H_{\phi}}=\frac{i v H_{m}^{(1)}(v r)}{\varepsilon k H_{m}^{(1) \prime}(v r)} .
$$

Introducing notations

$$
\begin{aligned}
& F_{3}(x)=Z_{L} H_{m}^{(1) \prime}(x)-A H_{m}^{(1)}(x), \\
& F_{4}(x)=Z_{L} H_{m}^{(2) \prime}(x)-A H_{m}^{(2)}(x),
\end{aligned}
$$

we scale the obtained expression for $Z_{\text {out }}$ with exponential function in order to handle finite metal conductivity:

$$
Z_{\text {out }}(r)=\frac{A H_{m}^{(2)}(v r) F_{3}\left(v r_{0}\right)-e^{2 i v\left(r-r_{0}\right)} H_{m}^{(1)}(v r) F_{4}\left(v r_{0}\right)}{H_{m}^{(2) \prime}(v r) F_{3}\left(v r_{0}\right)-e^{2 i v\left(r-r_{0}\right)} H_{m}^{(1) \prime}(v r) F_{4}\left(v r_{0}\right)},
$$

where $A=\frac{i v}{\varepsilon k}=i \sqrt{\frac{\mu}{\varepsilon}}$. 
The inward input impedance into the innermost region can be computed simply as:

$$
Z_{\text {in }}(r)=A \frac{J_{m}(v r)}{J_{m}^{\prime}(v r)} .
$$

For verification a test code (the same code as used for generating electromagnetic fields in [10]) based on a spectral domain Greens function for cylindrical geometry [11] was compared to the transverse resonance method for both TE and TM modes for the case $k_{z}=$ 0 . In this code, an excitation current in z-direction renders TM modes, and an excitation in transverse angular $\phi$ direction renders TE modes.

\subsection{TE110 and TE210 mode field distribution for the one-dimensional radial tomography}

In the case when the angular index $m \gg 1$ the electric field is dominant near resonator radius, see the equivalent field distribution for parallel plate TM disc resonators in [12]. Even TE210 mode has electric field significantly more confined near pipe radius compared to TE110 mode, which has a more homogeneous electric field distribution. This can be exploited tomographically, since TE210 mode field pattern penetrates less radially inwards than the TE110 mode. Thus, the TE210 mode is more sensitive to the presence of an outer concentric dielectric layer than the TE110 mode.

Having the quality factor and resonance frequency for each TE110 and TE210 mode, a set of four unknown material and dimensional parameters described in Table 1 can in theory be extracted using the same transverse resonance technique described previously.

\section{Open ended coaxial waveguide quarter-wave resonator probe}

An open ended coaxial waveguide quarter-wave resonator exposed to pipe can serve as an additional measurement probe, see Figure 2(left). If dimensioned properly (i.e. with suitable coaxial waveguide diameters $a$ and $b$ ), its penetration depth may be shorter than even the TE210 mode. The open-ended coaxial waveguide resonator gives typically a quality factor and resonance frequency for low-loss exposed media ("open-circuit" type load) as well as high loss exposed media ("short-circuit"). For the latter case, the frequency shift is negligible, while amplitude changes decreases with increased electric media loss. An intermediate region between low and high loss renders rather "arbitrary" wave-form, where the open end of the coaxial waveguide sees more of "matched load" impedance.

In this work, the response of the direct magnitude of the reflection coefficient $S_{11}$, see Figure 2(left), is minimized with respect to the model of an open ended coaxial waveguide and its quarter-wave transmission-line circuitry without any intermediate resonance frequency and Q-factor calculations. We apply the full-wave Hankel model as in [13]. If the pipe diameter is significantly larger than the outer diameter $b$ of the coaxial waveguide, the planar ground plane model used in [13] is assumed still to be valid. We note that the abrupt discontinuity from the coaxial waveguide section into a grounded plane excites higher order terms apart from the fundamental incident coaxial waveguide TEM mode. In [13], these higher modes are TM modes with only radial variations due to the angular symmetry. If the coaxial waveguide diameter $b$ increases relative to the pipe diameter $D$, the angular variations of the basis functions/higher modes would be stronger. However, they will be not as large as the radial higher mode excitations. A compromise in accuracy can be to assume an incident ideal TEM wave, matching the tangential electric 


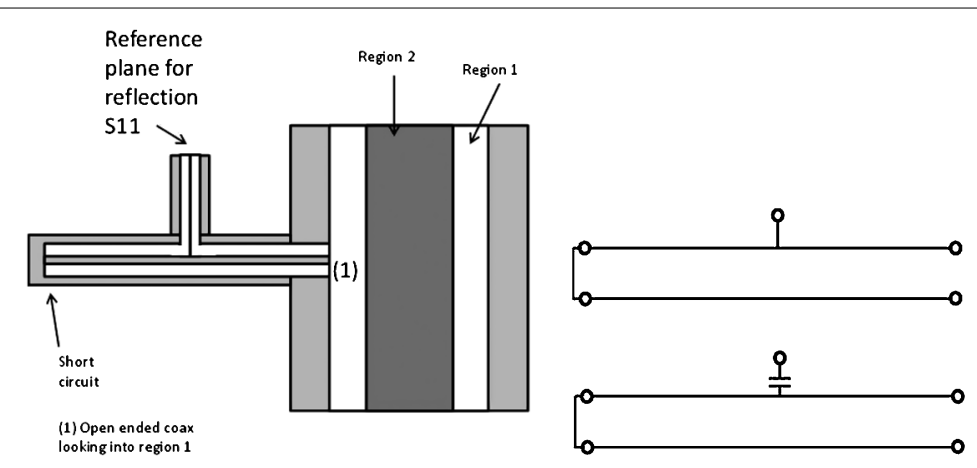

Figure 2 Model of the open ended galvanic coupled quarter-wave coaxial resonator with connected flush to a waveguide pipe section is presented on the left. Schematic circuits of galvanic and capacitive coupled quarter-wave resonators are shown on the right.

and magnetic fields at the curved open ended coaxial waveguide interface using a spectral domain approach [11], and using then basis functions/modes with angular dependency (in local open-ended coaxial waveguide coordinate system). For suitable coaxial waveguide dimensions $a, b$, a third characteristic, penetration depth which is smaller than in the TE210 mode, can be obtained. Thus, one can replace one of the four transverse resonance equations with an equation for the open-ended coaxial waveguide resonator.

As model for input impedance $Z_{\text {liq }}$, full-wave model of [13] is employed; using fundamental TEM mode, and two lowest TM modes as basis functions, the input impedance is calculated from the obtained reflection coefficient. In Figure 2(left) the reflection coefficient $S_{11}$ at the reference plane is obtained by the standard impedance transformations [8].

\subsection{Coupling to the open-ended quarter wave coaxial waveguide resonator}

By probing the resonator in the middle of the coaxial waveguide instead of at the left end, separation of the resonance frequencies is narrowed for the same physical resonator length. Practically (if not also theoretically) is impossible to select an optimal coupling to a quarter-wave open ended coaxial waveguide loaded with media when we have a large range of the real and imaginary parts of permittivity. We note that with salinities from 0 up to $25 \%$ the imaginary part of saline water changes with several orders of magnitude.

Experimentally we have found that using a pair of resonators as shown in Figure 2(right), a better sensitivity in amplitude and frequency shift is achieved. In this figure, the upper circuit represents a simply galvanic coupled quarter-wave resonator, while the lower figure shows capacitive coupled quarter-wave resonator. In this work we have used a coupling capacitance of $10 \mathrm{pF}$. We should note that a pair of capacitive and galvanic coupled resonators cannot render more information than a phase and magnitude reflection measurements taken directly at the open end of the coaxial waveguide. Rather, the resonator pair transforms the complex reflection data to the resonance type response, so that the benefit of both amplitude change as well as the frequency shift can be advantageous.

\section{Methods of reconstruction}

In this section we present methods of reconstruction of parameters presented in Table 1. We note that the four transverse resonance equations are minimized in the same manner 

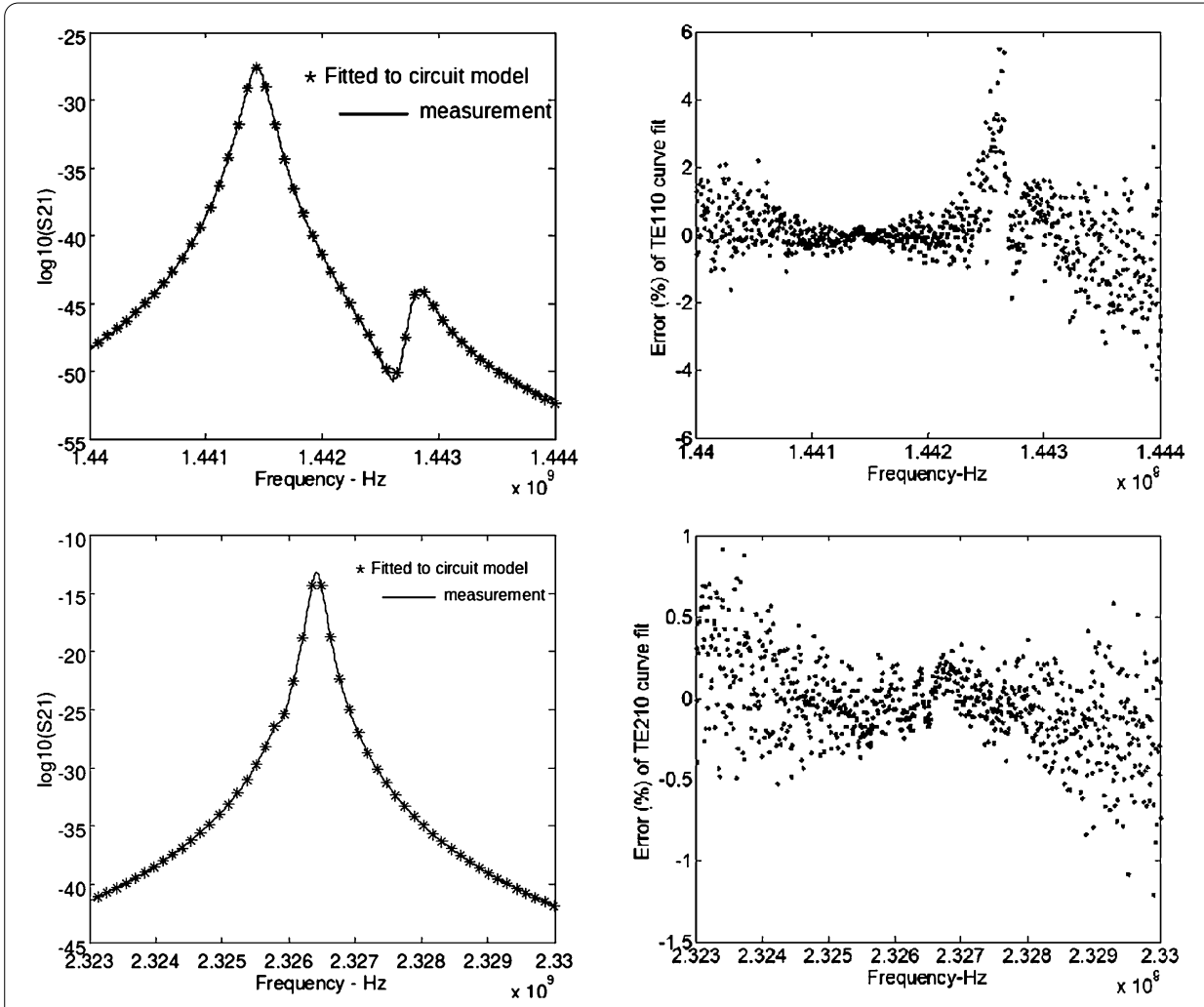

Figure 3 Result of step 1. Behavior of the Lorenzian function as a function of frequency, fitted to experimentally measured TE110 (left and right figures of the top figure) and a TE210 (left and right figures of the bottom figure) resonance in an air filled aluminium pipe of $128.5 \mathrm{~mm}$ internal diameter. Resonant frequencies and quality factors are extracted from a Lorentzian fit the transmission measurement. Complex resonant frequencies in (6) are calculated as $\omega_{0}=f_{0}^{\mathrm{TEm} 10}+i \frac{f_{0}^{\mathrm{TEm} 10}}{2 \mathrm{Q}_{0}^{\mathrm{TEm} 10}}$, which form input to the combined transverse resonant open ended coaxial waveguide functional. The top and bottom figures on the right side show the relative difference between the measured data and the Lorenzian function.

regardless parameters. Since we have four equations at hand and four unknowns, it is suitable to apply Newton's method.

The full computational scheme consists of two steps, see Figure 3 for results of the first step. First the resonance frequency and loaded quality factors are extracted for TE110 and TE210 modes. Typically, measured data is a transmission measurement. With weak coupling, the unloaded $Q$ factor can be approximated by the measured loaded $Q$ factor. Otherwise, a more sophisticated transfer function that models also the coupling circuitry must be applied to obtain the unloaded $Q$ factor and the resonance frequency.

Using results of work [2] we can conclude that for resonance spectrum data with signalto-noise ratio $<65$, a non-linear least squares fit to a Lorentzian curve is more accurate than fit to the phase versus frequency. Thus, for a sufficiently weakly coupled resonator under test, where unloaded $Q$ factor can be closer in value to the measured loaded $Q$ factor, the transmitted resonance spectrum is anticipated to have more noise simply due to the relatively low signal level. One may then conclude that non-linear least squares fit to a Lorentzian curve is suitable for characterizing weakly coupled resonator configurations. We refer to [2] for review of different methods for calculating of resonance spectrum, extraction of Q-factor and resonance frequency. The Lorentzian function is defined and 
Figure 4 The diagram shows the equivalent electric circuit for the transverse resonator.

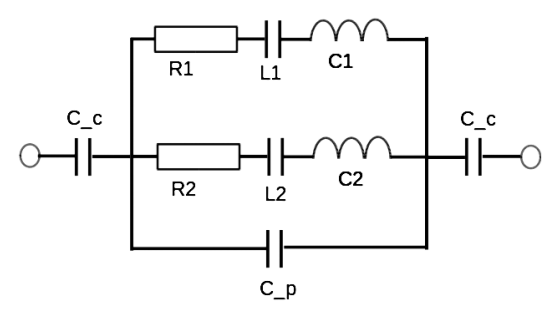

derived using a simple circuit analysis for circuit shown in Figure 4. We note that a realistic resonance curve has parasitic contributions from coupling circuitry, or parasitic parallel capacitance. Also, probes/antennas for resonance excitation may have intrinsic resonances which are "multiplied" to the resonance of interest.

Typically, resonance frequency $f_{0}$ and quality-factor $Q$ can be extracted by a simple peak search and a numerical direct extraction of the quality-factor from a ratio of band-width at half maximum peak value and resonance frequency. These extracted values are good initial guesses for $f_{\text {init }}^{\mathrm{TE} 110}, Q_{\text {init }}^{\mathrm{TE} 110}$ and $f_{\text {init }}^{\mathrm{TE} 210}, Q_{\text {init }}^{\mathrm{TE} 110}$. For one TE resonance, assuming that above conditions hold, we have four unknowns to be measured: amplitude $A m p_{0}^{\mathrm{TE} 110}, Q_{0}^{\mathrm{TE} 110}, f_{0}^{\mathrm{TE} 110}$ and $C_{0}^{\mathrm{TE} 110}$. The same is valid for TE210 resonance. Only $\mathrm{Amp}_{0}^{\mathrm{TE} 210}, f_{0}^{\mathrm{TE} 210}$ and $Q_{0}^{\mathrm{TE} 210}, C_{0}^{\mathrm{TE} 210}$ are needed to be measured in order to extract the four unknowns: liquid thickness, salinity, WLR and droplet ratio. The measured quality factors $Q_{0}^{\mathrm{TE} 110}$ and $Q_{0}^{\mathrm{TE} 210}$ as well as the measured resonance frequencies $f_{0}^{\mathrm{TE} 110}$ and $f_{0}^{\mathrm{TE} 210}$ can be approximated to the unloaded corresponding entities as long as the coupling to the resonator is sufficiently weak - otherwise, the unloaded quality factors and resonant frequencies must be calculated by an analysis that includes the coupling circuitry influence. In this work for simplicity we assume that the coupling is weak and that the unloaded and measured loaded entities are the same.

The second step of our computational procedure is to use the measured $Q$ factors and resonant frequencies for TE110 and TE210 modes as input to a combined transverse resonance condition for TE110 and TE210 modes, and solve then the obtained equations using the optimization algorithm. The reconstruction of the media and distribution of parameters is performed via minimization of the squared real and imaginary parts of transverse resonance functionals for TE110 and TE210 modes:

$$
\begin{aligned}
& J_{W}^{\operatorname{Re}}\left(f_{0}^{W}, Q_{0}^{W}, x, K\right)=\left(\operatorname{Re}\left(Z_{\text {in }}\left(f_{0}^{W}, Q_{0}^{W}, x, K\right)+Z_{\text {out }}\left(f_{0}^{W}, Q_{0}^{W}, x, K\right)\right)\right)^{2}=0, \\
& J_{W}^{\operatorname{Im}}\left(f_{0}^{W}, Q_{0}^{W}, x, K\right)=\left(\operatorname{Im}\left(Z_{\text {in }}\left(f_{0}^{W}, Q_{0}^{W}, x, K\right)+Z_{\text {out }}\left(f_{0}^{W}, Q_{0}^{W}, x, K\right)\right)\right)^{2}=0,
\end{aligned}
$$

where $W$ denote the TE110 or TE210 mode, $Z_{\text {out }}, Z_{\text {in }}$ are given by (32), (33) for TE mode and by (37), (38) for TM modes, $x=\left(h, R_{\mathrm{WLR}}, s, R_{\mathrm{DGR}}\right)$ is the vector of four unknowns described in Table 1 , and $K$ is the static input parameters (including temperature, pipe inner diameter and hydrocarbon permittivity). Having four unknowns and four equations, Newton-Raphson method is suitable for computations. One may add more sensors (the open ended coaxial waveguide resonator sensor shown in Figure 2, for instance) such that an overdetermined non-linear system of equations is obtained. This overdetermined system may be reduced back to a set of four equations either by adding the sensor functionals $J_{\text {quarter }}$ defined by (40) to the existing four transverse resonance functionals. It is also 
possible to replace one of the existing transverse resonance functionals with the sensor functional $J_{\text {quarter. }}$.

In this work, to obtain four unknown parameters $h, R_{\mathrm{WLR}}, s, R_{\mathrm{DGR}}$ we minimize the difference of the model value between the computed reflection coefficient $S_{11}$ (in magnitude) and the measured reflection coefficient $\tilde{S}_{11}$ (in magnitude) for two models: capacitive and galvanic. The measurements are taken exacly at the same point (reference plane) as the excitation (incident wave). A continuous wave in a certain frequency range is used, rather than a pulse. In addition to the four unknowns, the functional to be minimized also depends on the geometric dimensions and the dielectric media inside the pipe. Thus, our goal is to minimize the following functional:

$$
\begin{aligned}
J_{\text {quarter }}\left(h, R_{\mathrm{WLR}}, s, R_{\mathrm{DGR}}\right)= & \frac{1}{2} \int_{\omega_{1}}^{\omega_{2}}\left(\left|\mathrm{~S}_{11}^{\text {galv }}\left(h, R_{\mathrm{WLR}}, s, R_{\mathrm{DGR}}, a, b, \omega^{\prime}\right)\right|-\left|\tilde{S}_{11}^{\text {galv }}\left(\omega^{\prime}\right)\right|\right)^{2} d \omega^{\prime} \\
& +\frac{1}{2} \int_{\omega_{1}}^{\omega_{2}}\left(\left|S_{11}^{\mathrm{cap}}\left(h, R_{\mathrm{WLR}}, s, R_{\mathrm{DGR}}, a, b, \omega^{\prime}\right)\right|-\left|\tilde{S}_{11}^{\mathrm{cap}}\left(\omega^{\prime}\right)\right|\right)^{2} d \omega^{\prime} \\
& +\frac{1}{2} \alpha_{1}\left(h-h_{0}\right)^{2}+\frac{1}{2} \alpha_{2}\left(R_{\mathrm{WLR}}-R_{\mathrm{WLR}_{0}}\right)^{2} \\
& +\frac{1}{2} \alpha_{3}\left(s-s_{0}\right)^{2}+\frac{1}{2} \alpha_{4}\left(R_{\mathrm{DGR}}-R_{\mathrm{DGR}_{0}}\right)^{2}
\end{aligned}
$$

where $\alpha_{j}, j=1,2,3,4$ are small regularization parameters, such that $\alpha_{j} \in(0,1)$. They can be chosen as constant values depending on the noise level $\delta$, or iteratively using one of the iterative regularization algorithms, see $[14,15]$ for some of these algorithms. One of possible iterative choices for the computing of regularization parameters (see $[15,16]$ for computational details) is $\alpha_{j}^{n}=\alpha_{j}^{0}(n+1)^{-p}$, where $n$ is the number of iteration in any gradient-like method (in our case - the number of iteration in Newton's method), $p \in(0,1)$ and $\alpha_{j}^{0}$ are initial guesses for $\alpha_{j}, j=1, \ldots, 4$. Similarly with [17] we choose $\alpha_{j}=\delta^{\gamma}$, where $\delta$ is the noise level and $\gamma$ is a small number taken in the interval $(0,1)$. The values of $S_{11}^{\text {galv }}, S_{11}^{\text {cap }}$ are computed using the well-known formulas [8]

$$
S_{11}^{\text {galv }}=\frac{Z_{\text {tot }}^{\text {galv }}-Z_{0}}{Z_{\text {tot }}^{\text {galv }}+Z_{0}}, \quad S_{11}^{\text {cap }}=\frac{Z_{\text {tot }}^{\text {cap }}-Z_{0}}{Z_{\text {tot }}^{\text {cap }}+Z_{0}},
$$

where $Z_{\mathrm{tot}}^{\text {(galv,cap) }}$ is the total impedance at the reference plane at $Z_{0}=50.0 \mathrm{~m}$ for the galvanic and capacitive models, respectively. The integrals in (40) are approximated by a sum at the discrete measured frequency points $\omega_{i}^{\prime}$ with steps $\delta \omega^{\prime}$ :

$$
\begin{aligned}
J_{\text {quarter }}\left(h, R_{\mathrm{WLR}}, s, R_{\mathrm{DGR}}\right)= & \frac{1}{2} \sum_{i=0}^{N}\left(\left|S_{11}^{\text {galv }}\left(h, R_{\mathrm{WLR}}, s, R_{\mathrm{DGR}}, a, b, \omega_{i}^{\prime}\right)\right|-\left|\tilde{S}_{11}^{\text {galv }}\left(\omega_{i}^{\prime}\right)\right|\right)^{2} \cdot \delta \omega^{\prime} \\
& +\frac{1}{2} \sum_{i=0}^{N}\left(\left|S_{11}^{\mathrm{cap}}\left(h, R_{\mathrm{WLR}}, s, R_{\mathrm{DGR}}, a, b, \omega_{i}^{\prime}\right)\right|-\left|\tilde{S}_{11}^{\mathrm{cap}}\left(\omega_{i}^{\prime}\right)\right|\right)^{2} \cdot \delta \omega^{\prime} \\
& +\frac{1}{2} \alpha_{1}\left(h-h_{0}\right)^{2}+\frac{1}{2} \alpha_{2}\left(R_{\mathrm{WLR}}-R_{\mathrm{WLR}_{0}}\right)^{2} \\
& +\frac{1}{2} \alpha_{3}\left(s-s_{0}\right)^{2}+\frac{1}{2} \alpha_{4}\left(R_{\mathrm{DGR}}-R_{\mathrm{DGR}_{0}}\right)^{2} .
\end{aligned}
$$


Figure 5 Results of numerical simulations for exact values of parameters $s^{\text {true }}=10^{-4}, h^{\text {true }}$ $=1 \mathrm{~mm}, R_{\mathrm{DGR}}^{\text {true }}=10^{-2}, R_{\mathrm{WLR}}^{\text {true }}=0.1$. Top figures show error plots for four reconstructed parameters: stars denote errors for the model with using the open ended quarter resonator pair, and solid lines denote errors for the model without using the open ended quarter wave resonator. Bottom figure presents magnitude of reflections for capacitive (denoted by + ) and galvanic (denoted by solid line) models of the coupled open ended coaxial waveguide resonators.
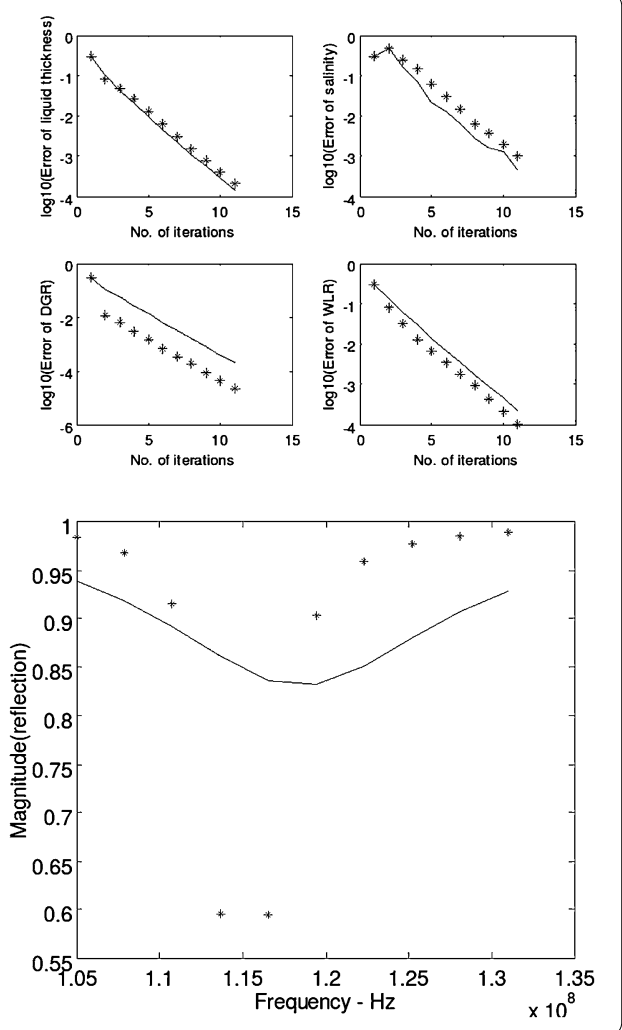

\section{Results of simulations}

In this section we present benchmarks on the set of simulations to extract parameters of Table 1. In all test cases, initial guesses in (41) were set to the following:

$$
\begin{aligned}
& s_{0}:=s^{\text {init }}=s^{\text {true }} \cdot 1.3, \quad R_{\mathrm{DGR}_{0}}:=R_{\mathrm{DGR}}^{\text {init }}=R_{\mathrm{DGR}}^{\text {true }} \cdot 0.7, \\
& R_{\mathrm{WLR}_{0}}:=R_{\mathrm{WLR}}^{\text {init }}=R_{\mathrm{WLR}}^{\text {true }} \cdot 0.7, \quad h_{0}:=R_{h}^{\text {init }}=h^{\text {true }} \cdot 1.3 .
\end{aligned}
$$

The frequency range for the pair of open ended coaxial waveguide resonator was adapted in order to keep the resonance within a sufficiently wide frequency range. In all our computations we have not added noise to the data. We present results of our computations on Figures 5-8. Using these figures we can conclude that the pair of open ended coaxial waveguide resonator significantly improves convergence and reduce the error defined as $e=\left|x_{\text {iterated }}-x_{\text {true }}\right| /\left|x_{\text {true }}\right|$ by a factor of 100 .

There is room for some optimization regarding the open ended coaxial waveguide resonator pair. For example, the coupling capacitance value could be further optimized. Number of frequency points and frequency range are other issues that may increase the benefits of having the open ended coaxial waveguide resonator pair. Also, for some combinations of salinity, WLR, DGR and liquid layer thickness, it may be more beneficial include either capacitive or galvanic coupled open ended coaxial waveguide resonator to increase convergence of the iterative procedure.

Regarding the coupling of the open ended coaxial waveguide resonator pair, it is noted that in the case of high salinity (typically salinity $>\sim 3 \%$ for $R_{\mathrm{WLR}}>0.5$ ) one can obtain better salinity sensitivity from the changes of amplitude with galvanic coupled resonator. 
Figure 6 Results of numerical simulations for exact values of parameters $s^{\text {true }}=10^{-4}, h^{\text {true }}$ $=1 \mathrm{~mm}, R_{\mathrm{DGR}}^{\text {true }}=10^{-3}, R_{\mathrm{WLR}}^{\text {true }}=0.1$. Top figures show error plots for four reconstructed parameters: stars denote errors for the model with using the open ended quarter resonator pair, and solid lines denote errors for the model without using the open ended quarter wave resonator. Bottom figure presents magnitude of reflections for capacitive (denoted by + ) and galvanic (denoted by solid line) models of the coupled open ended coaxial waveguide resonators.
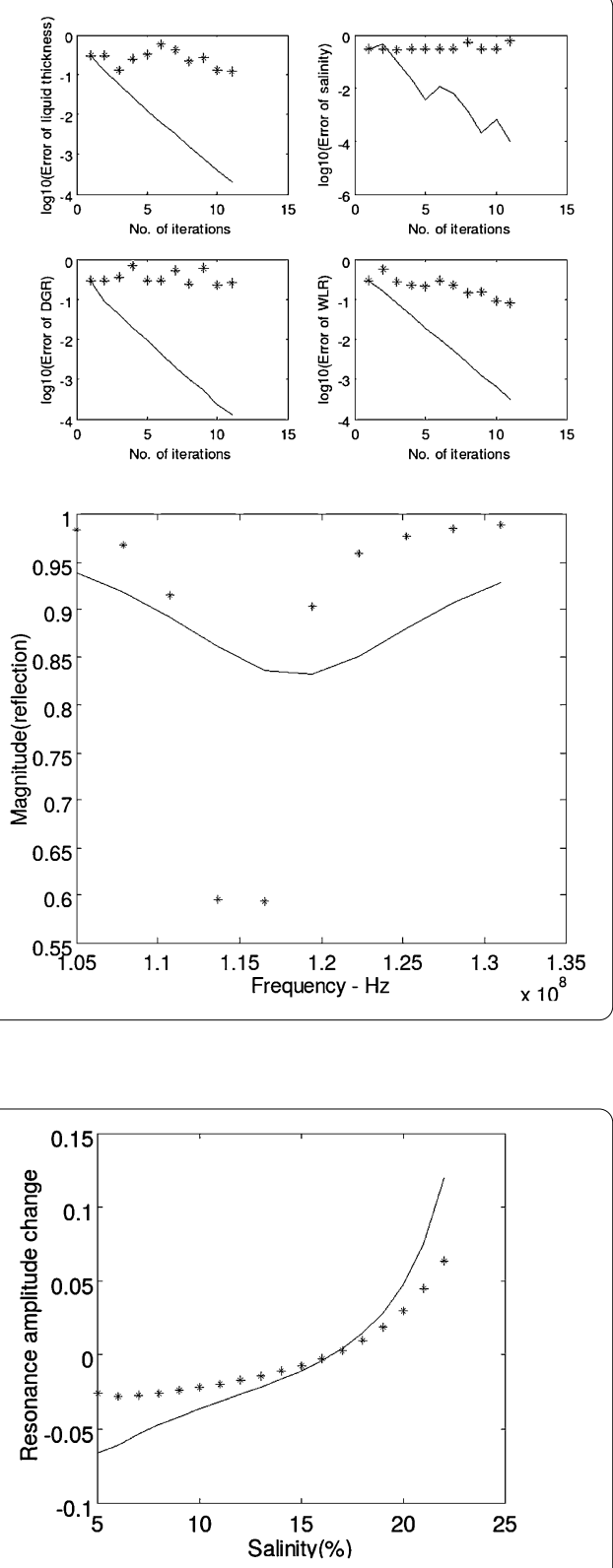

Figure 7 Behavior of resonance amplitude versus salinity at lowest resonance frequency. Crosses denote results for capacitive model while solid lines-for galvanic model of the coupled open ended coaxial waveguide resonator. Results are presented for liquid thickness $h=4 \mathrm{~mm}, R_{\mathrm{WLR}}=1, R_{\mathrm{DGR}}=10^{-5}$. The resonance amplitude is shown at the left figure and change in the resonance amplitude is presented at the right figure.

This is demonstrated on Figure 7. Here, we choose the liquid thickness $h=4 \mathrm{~mm}$ and $\mathrm{WLR}=1$. Using this figure we observe that the change of amplitude versus salinity (for salinities from $4 \rightarrow 22 \%$ ) is roughly by a factor two larger for the galvanic coupled resonator compared to the capacitive coupled resonator. Thus, the sensitivity of amplitude will be typically by a factor two larger for the galvanic coupled resonator except around the stagnation point at salinity $\sim 16 \%$. Note that the sensitivity is slightly depending on the coupling capacitance which in our computations is set to be $10 \mathrm{pF}$. 


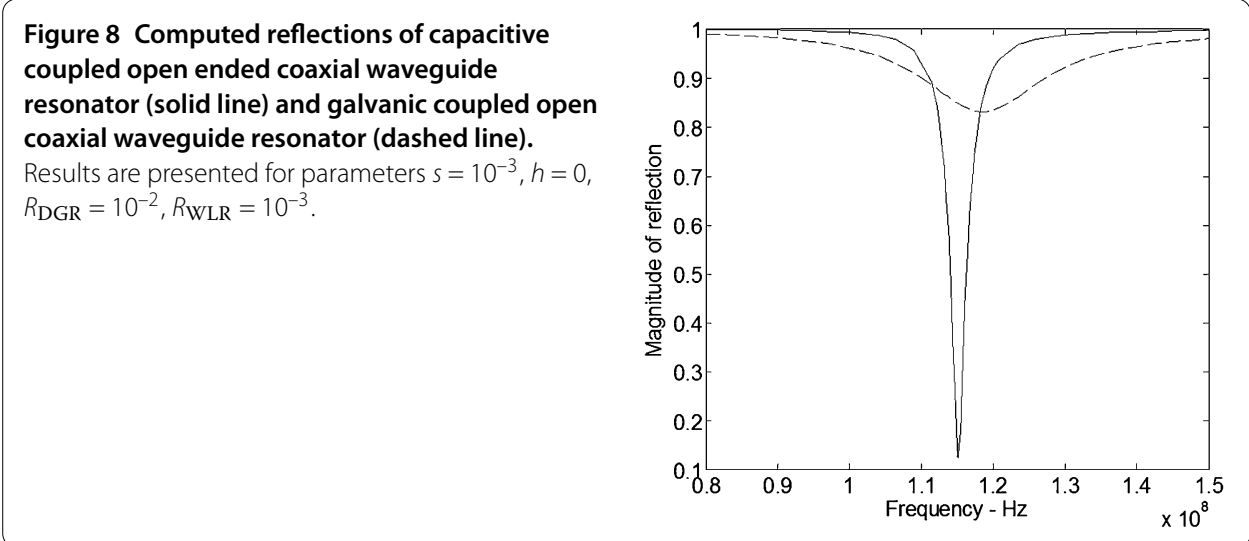

Table 2 The table shows the relative frequency change, by adding $0.5 \mu \mathrm{m}$ to the liquid film thickness $h$. Considering the $f_{0}$ listed in Table 3, these numbers represent frequency changes of the order $10^{2}-10^{3} \mathrm{~Hz}$

\begin{tabular}{lll}
\hline Mode & Relative tunability $\boldsymbol{h}=\mathbf{1} \mathbf{~ m m}$ & Relative tunability $\boldsymbol{h}=\mathbf{2} \mathbf{~ m m}$ \\
\hline TE1 10 & $3.701 \cdot 10^{-6}$ & $5.107 \cdot 10^{-6}$ \\
TE210 & $7.452 \cdot 10^{-6}$ & $1.293 \cdot 10^{-5}$ \\
Quarter wave & $1.520 \cdot 10^{-5}$ & $1.059 \cdot 10^{-5}$ \\
\hline
\end{tabular}

Table 3 The table shows the frequencies of the sensitivity analysed in Table 2

\begin{tabular}{lll}
\hline Mode & Frequency, $\boldsymbol{f}_{\mathbf{0}} \boldsymbol{h}=\mathbf{1} \mathbf{~ m m}$ & Frequency, $\boldsymbol{f}_{\mathbf{0}} \boldsymbol{h}=\mathbf{2} \mathbf{~ m m}$ \\
\hline TE1 10 & $1,041,453,582$ & $1,032,491,153$ \\
TE210 & $1,717,407,914$ & $1,684,125,223$ \\
Quarter wave, capacitive coupled & $112,188,018$ & $110,530,685$ \\
\hline
\end{tabular}

If we look at "low" salinity regime (salinity $0 \rightarrow \sim 3 \%$ for all WLR), the typical response of the capacitive coupled resonator is the one of a nearly critical coupled resonator with sharply defined skirts. On the other hand the galvanic coupled resonator has a much less pronounced resonance, see Figure 8 for simulated reflections computed as the ratio of the reflected voltage to the incident voltage. A change in liquid thickness or change in WLR would lead to a resonance frequency change and thus, the sensitivity of WLR or liquid thickness change is greater for the capacitive coupled resonator.

In Table 2 we show the shift of the resonance frequency for change in a small liquid thickness $\delta h=0.5 \mu \mathrm{m}$. In this table the relative tunability is computed as $\frac{f_{0}(h)-f_{0}(h+\delta h)}{f_{0}(h+\delta h)}$, where values of parameters are chosen as follows: $s=10^{-7}, R_{\mathrm{DGR}}=0, R_{\mathrm{WLR}}=1$. Using this table we observe that the TE210 mode is shifted twice as much compared to the TE110 mode. This is as expected, since electrical field in TE210 mode is weaker in the center of the spool. We also observe that the tunabilities for TE modes and quarter wave coaxial waveguide are of the same order of magnitude, even when the quarter-wave resonator tunability decreases with increased liquid layer $h$. Note that we observe opposite effect for TM modes (at least in the $h$-range used here).

\section{Discussion}

We have presented a full wave transverse resonance model for a circular cylindrical annular geometry. It was demonstrated numerically that four unknown physical parameters could be extracted. If we combine the transverse resonance model with the reflection data 
from open ended quarter-wave resonators, we may improve convergence and reduce the computational error by a factor of 100 .

Our numerical experiments demonstrate, that the combination of a galvanic and capacitive coupled open ended coaxial waveguide resonators renders higher sensitivity to determine WLR and liquid thickness. This is valid in low saline regime: for salinity $<\sim 3 \%$ in a water continuous liquid case or for any salinity where $R_{\mathrm{WLR}}<0.5$.

Using our experiments we can conclude that the improvement of the frequency sensitivity is due to capacitive coupled open ended coaxial waveguide resonators. For the high saline regime (water-continuous and salinity $>\sim 3 \%$ ), better sensitivity (in amplitude change due to change in salinity) is obtained using the galvanic coupled coaxial waveguide resonator.

\title{
Competing interests
}

The authors declare that they have no competing interests.

\section{Authors' contributions}

All authors have jointly worked to the manuscript. AE and TML implemented least squares solver and wrote draft of the manuscript. LB helped with mathematical statement of the problem and with writing the manuscript. All authors read and approved the final manuscript.

\section{Author details}

${ }^{1}$ Qamcom Research and Technology AB, Gothenburg, Sweden. ${ }^{2}$ Department of Mathematical Sciences, Chalmers University of Technology and University of Gothenburg, Gothenburg, 41296, Sweden. ${ }^{3}$ FMC Technologies, Kongsberg, Norway.

\begin{abstract}
Authors' information
AE is working as R\&D engineer at Qamcom Research and Technology AB, Gothenburg, Sweden, which provides services in the areas of signal processing, communication and radar systems. TML is working as R\&D engineer at FMC Technologies, Kongsberg Subsea AS P.O. Box 1012 N-3601-Kongsberg, Norway which develops equipment and technologies for exploration of offshore oil and gas fields. LB is Associate Professor at the Department of Mathematical Sciences at Chalmers University of Technology and Gothenburg University, SE-41296, Gothenburg, Sweden, with main research interest in the solution of coefficient inverse problems.
\end{abstract}

\section{Acknowledgements}

The authors are grateful for discussions with PS Kildal, Y Yang, Z Sipus, P Slättman, H Merkel and SP Hanserud. The work of LB is supported by the sabbatical program at the Faculty of Science, University of Gothenburg.

Received: 6 October 2016 Accepted: 23 February 2017 Published online: 11 April 2017

\section{References}

1. Eriksson A, Deleniv A, Gevorgian S. Orientation and direct current field dependent dielectric properties of bulk single crystal SrTiO3 at microwave frequencies. J Appl Phys. 2003;93:2848.

2. Petersan PJ, Anlage SM. Measurement of resonant frequency and quality factor of microwave resonators: comparison of methods. J Appl Phys. 1998;84:339.

3. Bruggeman DAG. Calculation of various physical constants of heterogeneous substances. Ann Phys. 1935;32(12).

4. Kiley EM, et al.Applicability study of classical and contemporary models for effective complex permittivity of metal powders. J. Microw. Power Electromagn Energy. 2012;46:26-38.

5. Gadani DH, et al.. Effect of salinity on the dielectric properties of water. Indian J Pure Appl Phys. 2012;50:405-10.

6. Lebedev NN. Special functions and their applications. New York: Dover Publications; 1972.

7. Harrington RF. Time-harmonic electromagnetic fields. Wiley: IEEE Press; 2001.

8. Collin RE. Foundations for microwave engineering. 2nd ed. Wiley: IEEE Press; 2000.

9. Eriksson A, Deleniv A, Gevorgian S. Resonant tunneling of microwave energy in thin film multilayer metal/dielectric structures. Microwave Symposium Digest, 2002 IEEE MTT-S International. 2002;3.

10. Beilina L, Eriksson A. Reconstruction of dielectric constants in a cylindrical waveguide. Springer Proceedings in Mathematics \& Statistics, Inverse Problems and Applications. 2015;120:97-109.

11. Šipuš Z, Kildal P-S, Leijon R, Johansson M. An algorithm for calculating Green's functions of planar, circular cylindrical, and spherical multilayer substrates. ACES Journal. 1998;13(3).

12. Eriksson A, Linner P, Gevorgian S. Mode chart of electrically thin parallel-plate circular resonators. IEEE Proceedings Microwaves Antennas and Propagation. 2001;1(148):51-5.

13. Baker-Jarvis J, et al. Analysis of an open-ended coaxial probe with lift-off for nondestructing testing. IEEE Transactions on Instrument and Measurement. 1994;43(5).

14. Tikhonov AN, Goncharsky AV, Stepanov WV, Yagola AG. Numerical methods for the solution of ill-posed problems. London: Kluwer; 1995

15. Bakushinsky A, Kokurin M, Smirnova A. Iterative methods for ill-posed problems. vol. 54. Berlin: De Gruyter; 2011. 
16. Malmberg JB, Beilina L. In: Iterative regularization and adaptivity for an electromagnetic coefficient inverse problem, AIP conference proceedings, ICNAAM2016. Rhodes, GREECE. 2016. p. 19.09-25.09.

17. Klibanov MV, Bakushinsky AB, Beilina L. Why a minimizer of the Tikhonov functional is closer to the exact solution than the first guess. J Inverse III-Posed Probl. 2011;19:83-105.

Submit your manuscript to a SpringerOpen ${ }^{\circ}$ journal and benefit from:

- Convenient online submission

Rigorous peer review

- Immediate publication on acceptance

- Open access: articles freely available online

- High visibility within the field

- Retaining the copyright to your article

Submit your next manuscript at springeropen.com 